\title{
A Middleware Platform for Intelligent Automation: An Industrial Prototype Implementation
}

\author{
Tiago Coito ${ }^{a}, *$, Miguel S.E. Martins ${ }^{a}$, Joaquim L. Viegas ${ }^{a}$, Bernardo Firme ${ }^{a}$, \\ João Figueiredo $^{\mathrm{b}}$, Susana M. Vieira ${ }^{\mathrm{a}}$, João M.C. Sousa ${ }^{\mathrm{a}}$ \\ a University of Lisbon, Instituto Superior Técnico, Department of Mechanical Engineering, Center of Intelligent Systems, IDMEC, Av. Rovisco Pais,1049-001 \\ Lisbon, Portugal \\ b University of Évora, Department of Mechatronics, IDMEC, Largo dos Colegiais 2, 7000-645 Évora, Portugal
}

\section{A R T I C L E I N F O}

\section{Article history:}

Received 13 March 2020

Received in revised form 23 July 2020

Accepted 23 September 2020

\section{Keywords:}

Intelligent Automation

Interoperability

Middleware

Fog computing

Data Preparation

Industry 4.0

\begin{abstract}
A B S T R A C T
The development of dynamic data-based Decision Support Systems (DSSs) along with the increasing availability of data in the industry, makes real-time data acquisition and management a challenge. Intelligent automation appears as a holistic combination of automation with analytics and decisions made by artificial intelligence, delivering smart manufacturing and mass customization while improving resource efficiency. However, challenges towards the development of intelligent automation architectures include the lack of interoperability between systems, complex data preparation steps, and the inability to deal with both high-frequency and high-volume data in a timely fashion. This paper contributes to industrial frameworks focused on the development of standardized system architectures for Industry 4.0, closing the gap between generic architectures and physical realizations. It proposes a platform for intelligent automation relying on a gateway or middleware between field devices, enterprise databases, and DSSs in real-time scenarios. This is achieved by providing the middleware interoperability, determinism, and automatic data structuring over an industrial communication infrastructure such as the OPC UA Standard over Time Sensitive Networks (TSN). Cloud services and database warehousing used to address some of the challenges are handled using fog computing and a multi-workload database. This paper presents an implementation of the platform in the pharmaceutical industry, providing interoperability and real-time reaction capability to changes to an industrial prototype using dynamic scheduling algorithms.
\end{abstract}

(C) 2020 Elsevier B.V. All rights reserved.

\section{Introduction}

Thanks to the decreasing sensor costs and the increasing computational power, the availability of data has been increasing in industry. The continuous data flow generated by the new industrial environments can be addressed by integrated analysis in the form of data-based DSSs. The idea of using machine intelligence to optimize the control of production processes and boost productivity is not new. Ohno, in the Toyota Production System, called it autonomation or "automation with a human touch" (Ohno 1988). Today, the Industry 4.0 initiative, fourth industrial revolution, digital manufacturing or smart factories all have in common the organizational

\footnotetext{
* Corresponding author

E-mail addresses: tiagoascoito@tecnico.ulisboa.pt (T. Coito), miguelsemartins@tecnico.ulisboa.pt (M.S.E. Martins),

joaquim.viegas@tecnico.ulisboa.pt (J.L. Viegas), bernardo.firme@tecnico.ulisboa.pt (B. Firme), jfig@uevora.pt (J. Figueiredo), susana.vieira@tecnico.ulisboa.pt (S.M. Vieira),jmsousa@tecnico.ulisboa.pt (J.M.C. Sousa).
}

changes introduced by the adoption of technologies and devices, capable of autonomously interact with one another, and with people, along the value of chain (Kagermann et al., 2013). In this new autonomous data-driven industry, Intelligent Automation appears as a concept that aggregates all the digital technologies through the combination of automation with analytics and decisions made by artificial intelligence. The potential and growth of these systems are pressuring the industry towards delivering cheaper and more personalized products. Mass customization is the effort of combining both the low costs of mass production with the flexibility of custom production. Companies are forced to produce smaller and more diverse batches, making the level of organization, integration, and communication more complex and both real-time, and decentralized-decision dependent (McFarlane et al. 2013). Within this context, data has become a valuable business asset.

If knowledge is power, communication is paramount. The ability to grow is linked to the efficiency offered and the flexibility with which new systems plug-and-work with previous, whilst taking advantage of global standards. The acquisition, manipulation and 